\title{
Decreased level of irisin, a skeletal muscle cell-derived myokine, is associated with post-stroke depression in the ischemic stroke population
}

Wen-Jun Tu ${ }^{1,2,3 \dagger}$, Han-Cheng Qiu ${ }^{2 \dagger}$, Qiang Liu ${ }^{1,4^{*}}$, Xuemei Li ${ }^{3}$, Ji-Zong Zhao ${ }^{2,5^{*}}$ and Xianwei Zeng ${ }^{3}$

\begin{abstract}
Background: Depression is a frequent mood disorder in stroke patient. Our aim was to determine irisin levels in serum and investigate their associations with post-stroke depression (PSD) in a 6-month follow-up study in Chinese patients with first-ever acute ischemic stroke (AIS).

Methods: The subjects were first-ever AIS patients who were hospitalized at three stroke centers during the period from January 2015 to December 2016. Neurological and neuropsychological evaluations were conducted at the 6-month follow-up. Serum irisin concentrations were measured by enzyme-linked immunosorbent assay (ELISA).

Results: During the study period, 1205 patients were included in the analysis. There were 370 patients (30.7\%) classified as depression. The depression distribution across the irisin quartiles ranged between $49.8 \%$ (first quartile) and $9.9 \%$ (fourth quartile). In the patients with depression, serum irisin levels were lower compared with those in patients without depression $(P<0.001)$. In a multivariate model using the first (Q1) quartile of irisin vs. Q2-4 together with the clinical variables, the marker displayed predictive information and increased risk of PSD by $75 \%$ (odds ratio [OR] for Q1, 1.75 [95\% confidence interval [Cl], 1.15-2.65]). In addition, a model containing known risk factors plus irisin compared with a model containing known risk factors without irisin showed a greater discriminatory ability; the area under the curve (AUC) increased from 0.77 to 0.81 ( $95 \% \mathrm{Cl}, 0.76-0.86)$.
\end{abstract}

Conclusions: The data suggested that reduced serum levels of irisin were powerful biological markers of risk of developing PSD even after adjustment by variables. Further studies are necessary to confirm this association, which may open the way to the proposal of new therapeutic options.

Trial registration: ChiCTR-OPC-17013501. Retrospectively registered 23 September 2017

Keywords: Irisin, Depression, Acute ischemic stroke, Chinese

\section{Background}

Stroke is a medical emergency caused by interrupted blood supply to the brain that further leads to rapid loss of brain functions. It is the second leading cause of death worldwide and associated with long-term disability [1]. Post-stroke depression (PSD) is a frequent mood

\footnotetext{
* Correspondence: liuqiang_cams@163.com; zhaojztj205@163.com ${ }^{\dagger}$ Equal contributors

'Institute of Radiation Medicine, China Academy of Medical Science and Peking Union Medical College, Tianjin, China

${ }^{2}$ Department of Neurosurgery, Beijing Tiantan Hospital of Capital Medical University, Beijing, China

Full list of author information is available at the end of the article
}

disorder in stroke patient. It worsened stroke-related outcomes in the form of greater functional disability and higher mortality [2]. Hadidi et al. [3] suggested that patients with PSD shown far less recovery from functional impairments compared with no depression patients with stroke and were 3.4 times more likely to die during the first 10 years after stroke.

Irisin is a small polypeptide hormone that is cleaved from the fibronectin type III domain containing 5 (FNDC5) [4]. The secreted level of irisin is commonly increased by exercise [5]. The highest basal levels of FNDC5 expression are seen in the brain and heart, with low basal levels in

(c) The Author(s). 2018 Open Access This article is distributed under the terms of the Creative Commons Attribution 4.0 International License (http://creativecommons.org/licenses/by/4.0/), which permits unrestricted use, distribution, and 
the liver, lung, skeletal muscle, and testis [6]. Irisin might be the master hormone to decide whether energy is released as heat or stored as ATP.

Accumulating evidence has demonstrated that irisin contributes to the regulation of glucose and lipid metabolism in the skeletal muscle and adipose tissue [7, 8]. Besides, irisin was also found to have an essential role in the chronic kidney disease [9], obesity [10], insulin resistance [11], or type 2 diabetes [12]. Zhang et al. [13] observed that physical exercise may signal to the brain to modulate metabolic activity and locomotion via irisin. Furthermore, irisinencoding gene (FNDC5) variant can change blood pressure in men with type 2 diabetes [14], while irisin improves endothelial function in type 2 diabetes [15] and in a mouse model of obesity [16]. In addition, irisin protects against endothelial injury and ameliorates atherosclerosis in Apo-E knockout mice [17]. Due to the crosstalk between metabolic dysfunction and cardio-cerebrovascular diseases, thus, a role for irisin in the cardio-cerebrovascular system is expected.

Furthermore, irisin plays substantial roles in the pathophysiology of metabolic diseases [18]. Metabolic diseases are associated with depression and depressive symptoms $[19,20]$. Moreover, irisin has been found to be not only a myokine but also an adipokine [21]. Evidences suggest that adipokines are involved in depression pathophysiology [22]. Wang and Pan [23] had demonstrated that irisin has a crucial role in inducing antidepressant-like effects in chronic unpredictable stress (CUS) rats by regulating energy metabolism in the prefrontal cortex of the brain.

A previous meta-analysis found that exercise was an evidence-based treatment for depression [24], and this effect has been linked to a PGC-1 $\alpha$-FNDC5/irisin pathway, which is activated by exercise in the hippocampus in mice and induces a neuroprotective gene program [25]. Furthermore, a previous study showed that a low serum irisin level was a predictor of poor early functional outcome in ischemic stroke patients [26]. We hypothesized that irisin serum level is a risk factor for depression among patients with ischemic stroke. The aim of this study is to determine irisin levels in serum and investigate their associations with PSD in a 6-month follow-up study in Chinese patients with first-ever acute ischemic stroke (AIS).

\section{Methods}

\section{Study population}

The subjects were first-ever AIS patients who were hospitalized at three stroke centers (Beijing, Weifang, and Wuhan) during the period from January 2015 to December 2016. AIS was defined according to the World Health Organization Multinational Monitoring of Trends and Determinants in Cardiovascular Disease (WHO-MONICA) criteria and was verified by magnetic resonance imaging (MRI) reports performed within $24 \mathrm{~h}$ after admission. Exclusion criteria were as follows: (1) pre-stroke diagnosis of dementia or cognitive impairment, a history of any psychiatric illness, and decreased level of consciousness; (2) malignant tumor, sarcopenia, liver insufficiency and renal insufficiency (creatinine > $1.5 \mathrm{mg} / \mathrm{dL}$ ), metabolic abnormalities (not included diabetes), severe edema, and autoimmune diseases; (3) used psychotropic drugs prior to stroke onset; (4) without informed consent, lost blood samples, or current medications used that influence serum irisin levels; and (5) death during the follow-up.

The control group consisted of 120 healthy volunteers (sex and age matched) without a history of psychiatric or neurological disorders. The control group also received clinical assessment, and their score on the Hamilton Scale was less than 7 . Written informed consent were obtained from all patients, and this study conformed to the principles of the Declaration of Helsinki and was approved by the investigational review board of the Weifang Medical University.

\section{Clinical variables and follow-up}

At baseline, demographic data (age, sex, and body mass index $[\mathrm{BMI}]$ ) and the following vascular risk factors, hypertension, diabetes mellitus, hypercholesterolemia, smoking, and a family history of ischemic stroke, were collected. Pre-stroke therapy (oral anticoagulants or antiplatelet agents) and acute treatment (IV thrombolysis and/or mechanical thrombectomy) were also recorded. Patients were evaluated with the National Institute of Health Stroke Scale (NIHSS) [27] score at their admission, performed by a stroke neurologist certified. Stroke subtype was classified according to TOAST (Trial of Org 10172 in Acute Stroke Treatment) criteria. Brain imaging (MRI) was done routinely within $24 \mathrm{~h}$ after admission. MRI with diffusion-weighted imaging (DWI) was available for some patients. The infarct volume was calculated by using the formula $0.5 \times a \times b \times c$ (where $a$ is the maximal longitudinal diameter, $b$ is the maximal transverse diameter perpendicular to $a$, and $c$ is the number of $10-\mathrm{mm}$ slices containing infarct) [28].

The end point was psychological evaluation on month 6 after admission. Psychological evaluation was performed by the trained psychologist at 6 months. All patients were interviewed using the structured clinical interview of DSM-IV (SCID-I-R) [29], allowing for a diagnosis of both major and minor depression. Inter-rater reliability was examined in 100 patients, and the kappa was 0.92. The severity of depressive symptoms was measured with the 17-item Hamilton Depression Rating Scale (HAM-D) [30]. We have used the validated version for the Chinese population. Data concerning demographics, marital status, living situation, family history of psychiatric disorders, 
and drug treatment were also collected by interview. Functional outcome was also obtained according to the Modified Rankin Scale (mRS) score [31]. The evaluation was conducted by a neurologist/psychiatrist who was unaware of the type, size, and location of the index stroke at the time of the investigation and throughout the diagnostic procedure. In addition, specific details about physical activity in some patients $(n=223)$ were assessed by a validated questionnaire adapted from the Canadian Community Health Survey [32].

\section{Blood collection and laboratory test}

All blood samples were collected on the first day of admission under fasting state. Blood samples were centrifuged at $1000 \times g$ for $12 \mathrm{~min}$, and the serum was separated and stored at $-80{ }^{\circ} \mathrm{C}$ until the time of assay. Biochemical measurements were done using standard laboratory methods. The levels of serum total cholesterol (TC), triglycerides (TG), high-sensitivity C-reactive protein(Hs-CRP), glucose, homocysteine ( $\mathrm{HCY})$, and high-density lipoprotein cholesterol (HDL-C) were measured by enzymatic assays, and fasting glucose levels were measured using the hexokinase method (Autoanalyzer Model 7600 II; Hitachi, Tokyo, Japan). Insulin resistance (IR) was estimated from fasting serum measurements using the homeostasis model assessment of insulin resistance (HOMA-IR): insulin $(\mu \mathrm{IU} / \mathrm{mL}) \times$ glucose $(\mathrm{mmol} / \mathrm{L}) / 22.5$. Serum irisin concentrations were measured in duplicate by using the enzyme-linked immunosorbent assay (ELISA) kits (Code No. SK00170-09; Aviscera Biosciences, Santa Clara, CA, USA), in accordance with the manufacturer's instructions. The sensitivity of the assay was $1.0 \mathrm{ng} / \mathrm{mL}$, and the linear range of the standard was $1-500 \mathrm{ng} / \mathrm{mL}$. The intra-and inter-assay coefficients of variation $(\mathrm{CV})$ were $3.3-5.0 \%$ and $4.3-6.8 \%$, respectively. Serum interleukin-6 (IL-6) was determined by ELISA (Human IL-6 Quantikine ELISA Kit [Code No. D6050], R\&D Systems, Inc., Minneapolis, MN, USA). The sensitivity of the assay was $0.7 \mathrm{pg} / \mathrm{mL}$, and the linear range of the standard was $3-300 \mathrm{pg} / \mathrm{mL}$. The intra- and inter-assay CV were $1.7-4.4 \%$ and $3.8-6.4 \%$, respectively. Serum levels of serotonin were quantitatively determined via direct competitive radioimmunoassay (RIA) with the separation of bound/free ligands using an IgG antibody (ALPCO Diagnostics, Salem, NH 03079, USA). The manufacturer's instructions were followed. The results were reported in nanograms per milliliter. The analytical sensitivity of this serotonin RIA is $0.3 \mathrm{ng} / \mathrm{mL}$ with the $\mathrm{CV}$ for the intra- and inter-assay reproducibility as $2.5-4.7$ and $3.6-5.8 \%$, respectively. For all measurements, the levels that were not detectable were considered to have a value equal to the lower limit of detection of the assay. Determinations were performed in an independent laboratory blinded to clinical and neuroimaging data.

\section{Statistical analysis}

Results are expressed as percentages for categorical variables and as means (standard deviation, S.D.) or medians (quartiles) for the continuous variables, depending on the normal or non-normal distribution of data. Proportions were compared using the $\chi^{2}$ test, and Student's $t$ test and analysis of variance (ANOVA) were employed for the normally distributed variables, while the MannWhitney $U$ test was employed for the asymmetrically distributed variables. Spearman's rank correlation was used for bivariate correlations.

The relationship between serum levels of irisin and PSD were evaluated using univariate and multivariate regression analyses. We used crude models and multivariate models adjusted for all significant predictors and report odds ratios (ORs). For multivariate analysis, categorical variables included age, sex, BMI, stroke etiology, the NIHSS score, infarct volume, pre-stroke and acute treatment, vascular risk factors, mRS at follow-up, family history of psychiatric disorders, lesion location, living with offspring, widowhood, HOMA-IR, blood levels of glucose, Hs-CRP, HCY, IL-6, TG, TC, HDL, LDL, and serotonin and irisin quartiles. For a more detailed exploration of the irisin and PSD, we also used multivariate analysis models to estimate adjusted OR and 95\% CIs of PSD for irisin quartiles (with highest irisin quartile as reference). In addition, serum irisin level was also dichotomized according to irisin quartiles, and the grouping of quartiles 2-4 was defined as normal while the grouping of quartile 1 as low.

Second, receiver operating characteristic (ROC) curves was used to test the overall predict accuracy of irisin and other markers to diagnose PSD, and results were reported as area under the curve (AUC). Integrated discrimination improvement (IDI) and net reclassification improvement (NRI) indices were calculated to determine the clinical utility of the addition of irisin to established risk factors and the ability of irisin to improve PSD prediction [33].

Finally, the sensitivity analysis was performed to test the robustness of the results, and only those patients with information about physical activity were included. In multivariate logistic regression analysis, we calculated the OR of irisin level to predict PSD after adjusted physical activity and other risk factors. All statistical analyses were performed with SPSS for Windows, version 21.0 (SPSS Inc., Chicago, IL, USA), the ROCR package (version 1.0-2), and the GraphPad Prism 5.0. Statistical significance was defined as $P<0.05$.

\section{Results}

\section{Baseline characteristics of study samples}

The study cohort consisted of 1710 patients at stroke admission. By the time of 6-month follow-up, 325 had passed away, 130 declined the invitation to participate, 
and 50 had lost follow-up, leaving 1205 individuals (Fig. 1). However, these included patients were similar in terms of baseline characteristics [age $(P=0.18)$, sex $(P=0.69)$, and BMI $(P=0.42)]$ compared to the overall cohort. Overall, the median age was 65 (IQR, 56-75), and 640 (53.1\%) were male. The irisin levels were obtained in stroke patients with a median value of $95.9 \mathrm{ng} / \mathrm{mL}$ (IQR, 71.1$144.0 \mathrm{ng} / \mathrm{mL}$ ), which was significantly lower than the levels in controls (137.5; IQR, 100.4-171.5 ng/mL). The baseline characteristics of the 1205 stroke patients with and without PSD were described in Table 1.

\section{Main findings}

During the 6-month follow-up period, all surviving patients were examined regarding depression. There were 370 patients $(30.7 \%$, 95\% CI $28.1-33.3 \%)$ classified as depression. The depression distribution across the irisin quartiles ranged between $49.8 \%$ (first quartile) to $9.9 \%$ (fourth quartile) (Fig. 2). Patients with depression were older and more frequently were widowhood, living with offspring, higher initial stroke severity, larger infarct volume, higher serum levels of Hs-CRP and HCY, and lower levels of serotonin (Table 1).

Serum levels of irisin decreased with increasing severity of stroke as defined by the NIHSS score. There was a negative correlation between levels of irisin and the NIHSS $(r$ spearman] $=-0.343, P<0.001)$. There were modest correlations between levels of irisin and Hs-CRP $(r=-0.232, P<0.001)$, IL-6 $(r=-0.287, P<0.001)$, HCY $(r=-0.269, P<0.001)$, serotonin $(r=0.159, P=0.12)$, and HDL $(r=0.188, P=0.001)$. In addition, there is a negative correlation between levels of irisin and BMI $(r=-0.273$, $P<0.001)$. There was no correlation between levels of irisin and sex $(P=0.24)$ and age $(P=0.11)$. Furthermore, there was a modest negative correction between irisin and HAM-D score $(r=-0.211, P<0.001)$. There was still a significant negative correction between irisin serum levels and HAM-D score, using ordered logistic regression after multivariate adjustment for possible confounders $(P=0.003)$.

In the 370 patients with depression, serum irisin levels were lower compared with those in patients without depression [74.2 (IQR, 60.4-108.4) ng/mL vs. 120.3 (IQR, 77.6-164.8) ng/mL; $P<0.001$; Fig. 3]. In univariate logistic regression analysis, we calculated the odds ratio (OR) of irisin level to predict PSD as compared with the NIHSS score and other risk factors. With an unadjusted OR of 0.981 (95\% CI, 0.974-0.989; $P<0.001$ ), irisin had a strong association with PSD. After adjusting for all other significant outcome predictors, irisin remained an independent PSD predictor with an adjusted OR of 0.989 (95\% CI, 0.985-0.995). In multivariate models comparing the first $(\mathrm{Q} 1)$, second $(\mathrm{Q} 2)$, and third $(\mathrm{Q} 3)$ quartiles against the fourth (Q4) quartile of irisin (Table 2),

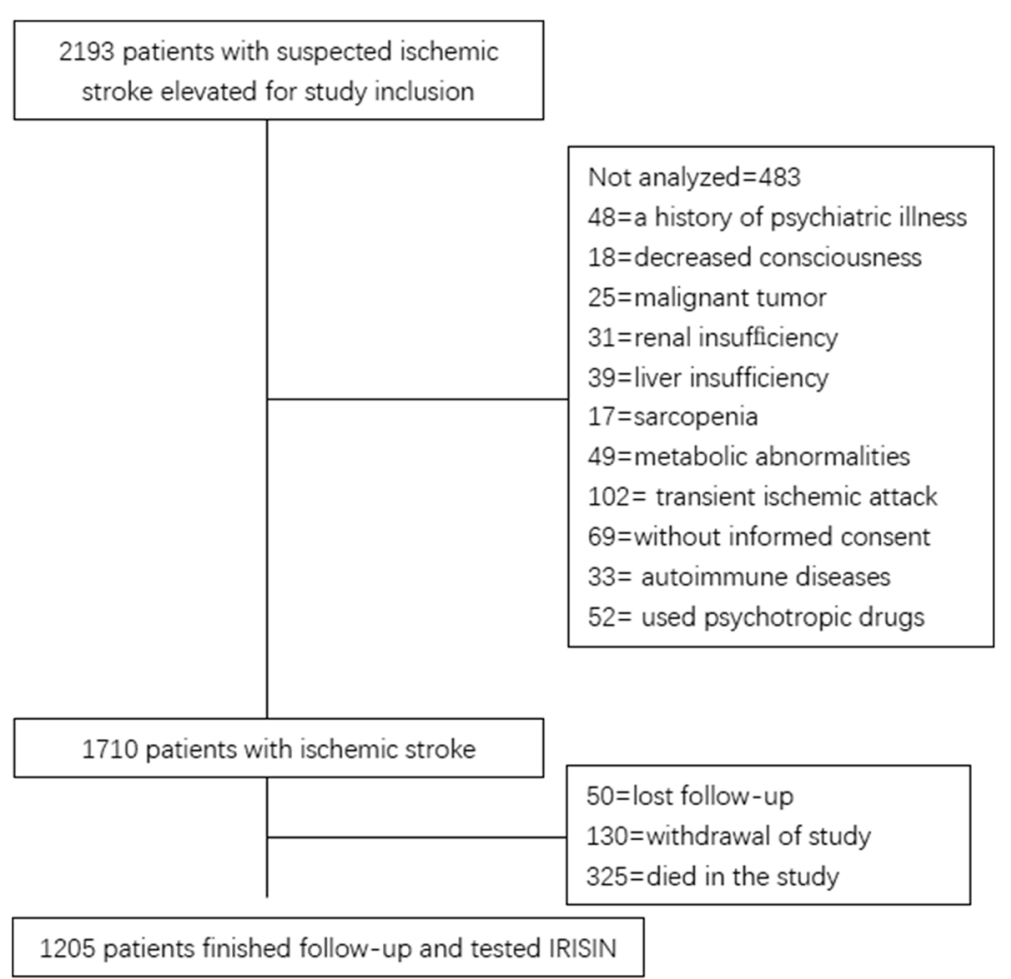

Fig. 1 Study profile/flow sheet of the study 
Table 1 Baseline clinical characteristics in patients with and without post-stroke major depression at 6 months

\begin{tabular}{|c|c|c|c|}
\hline & No depression & Depression & $P$ \\
\hline N & 835 & 370 & - \\
\hline Age (years), median (IQR) & $64(56-75)$ & $69(57-78)$ & 0.001 \\
\hline Male, $n(\%)$ & $430(51.5)$ & $210(56.8)$ & 0.450 \\
\hline BMI $\left(\mathrm{kg} / \mathrm{m}^{2}\right)$, median (IQR) & $26.8(24.2-28.7)$ & $27.2(24.3-28.8)$ & 0.312 \\
\hline Stroke severity, median NIHSS score (IQR) & $6(3-9)$ & $11(6-16)$ & 0.005 \\
\hline Lesion volumes (mL), median (IQR) & $32(7-61)$ & $48(14-75)$ & 0.009 \\
\hline mRS at 3 months, median (IQR) & $1(0-2)$ & $1(1-2)$ & 0.252 \\
\hline \multicolumn{4}{|l|}{ Vascular risk factors (\%) } \\
\hline Hypertension & 67.1 & 70.2 & 0.683 \\
\hline Diabetes mellitus & 34.1 & 38.3 & 0.597 \\
\hline Coronary heart disease & 31.1 & 34.0 & 0.706 \\
\hline Family history for stroke & 21.0 & 25.5 & 0.503 \\
\hline Current smoking & 22.2 & 23.4 & 0.856 \\
\hline \multicolumn{4}{|l|}{ Pre-stroke treatment (\%) } \\
\hline Antiplatelet agents & 31.7 & 34.0 & 0.765 \\
\hline Anticoagulants & 21.0 & 23.4 & 0.718 \\
\hline \multicolumn{4}{|l|}{ Endovascular/surgical revascularization (\%) } \\
\hline Family history of psychiatric disorders (\%) & 10.2 & 27.7 & 0.002 \\
\hline Widowhood (\%) & 9.0 & 25.5 & 0.003 \\
\hline Living with offspring (\%) & 10.2 & 27.7 & 0.002 \\
\hline Stroke etiology (\%) & & & 0.623 \\
\hline Atherothrombotic & 32.9 & 29.8 & \\
\hline Cardioembolic & 28.7 & 25.5 & \\
\hline Lacunar & 16.8 & 23.4 & \\
\hline Unknown & 21.6 & 21.3 & \\
\hline Lesion location (\%) & & & 0.371 \\
\hline Frontal & 26.9 & 21.3 & \\
\hline Parietal & 14.4 & 19.1 & \\
\hline Basal ganglia & 20.4 & 21.3 & \\
\hline Posterior fossa & 17.4 & 21.3 & \\
\hline Other & 21.0 & 17.0 & \\
\hline \multicolumn{4}{|l|}{ Laboratory findings, median (IQR) } \\
\hline Glucose $\left(\mathrm{mmol} \mathrm{L}^{-1}\right)$ & $6.18(5.54-6.75)$ & $6.22(5.58-6.85)$ & 0.206 \\
\hline $\mathrm{Hs}-\mathrm{CRP}\left(\mathrm{mg} \mathrm{dL}^{-1}\right)$ & $0.48(0.24-0.69)$ & $0.75(0.46-1.23)$ & 0.009 \\
\hline $\mathrm{HCY}\left(\mathrm{mmol} \mathrm{L}{ }^{-1}\right)$ & $13.8(9.6-16.5)$ & $17.2(12.1-21.6)$ & 0.018 \\
\hline $\mathrm{TC}\left(\mathrm{mmol} \mathrm{L}{ }^{-1}\right)$ & $4.86(4.32-5.36)$ & $4.90(4.32-5.41)$ & 0.428 \\
\hline $\mathrm{TG}\left(\mathrm{mmol} \mathrm{L}{ }^{-1}\right)$ & $1.89(1.43-2.21)$ & $1.88(1.40-2.26)$ & 0.501 \\
\hline $\mathrm{HDL}-\mathrm{C}\left(\mathrm{mmol} \mathrm{L}{ }^{-1}\right)$ & $1.16(1.03-1.24)$ & $1.18(1.05-1.29)$ & 0.332 \\
\hline LDL-C (mmol L $\left.{ }^{-1}\right)$ & $2.48(2.11-2.76)$ & $2.40(2.04-2.83)$ & 0.242 \\
\hline IL-6 (pg mL $\left.{ }^{-1}\right)$ & $101.2(76.5-138.6)$ & $132.2(102.3-164.8)$ & 0.003 \\
\hline HOMA-IR & $2.13(1.52-3.04)$ & $2.20(1.62-3.11)$ & 0.151 \\
\hline Serotonin $\left(n g \mathrm{~mL}^{-1}\right)$ & $1.65(0.39-3.83)$ & $1.18(0.35-2.96)$ & $<0.001$ \\
\hline Irisin $\left(n g \mathrm{~mL}^{-1}\right)$ & $120.3(77.6-164.8)$ & $74.2(60.4-108.4)$ & $<0.001$ \\
\hline
\end{tabular}

Results are expressed as percentages for categorical variables and as medians (quartiles) for the continuous variables NIHSS National Institutes of Health Stroke Scale, mRS Modified Rankin Scale, S.D. standard deviation, IQR interquartile range, Hs-CRP high-sensitivity C-reactive protein, $H C Y$ homocysteine, $B M I$ body mass index, $H D L-C$ high-density lipoprotein cholesterol, $L D L-C$ low-density lipoprotein cholesterol, $T G$ triglyceride, $T C$ total cholesterol, HOMA-IR homeostatic model of assessment insulin resistance 


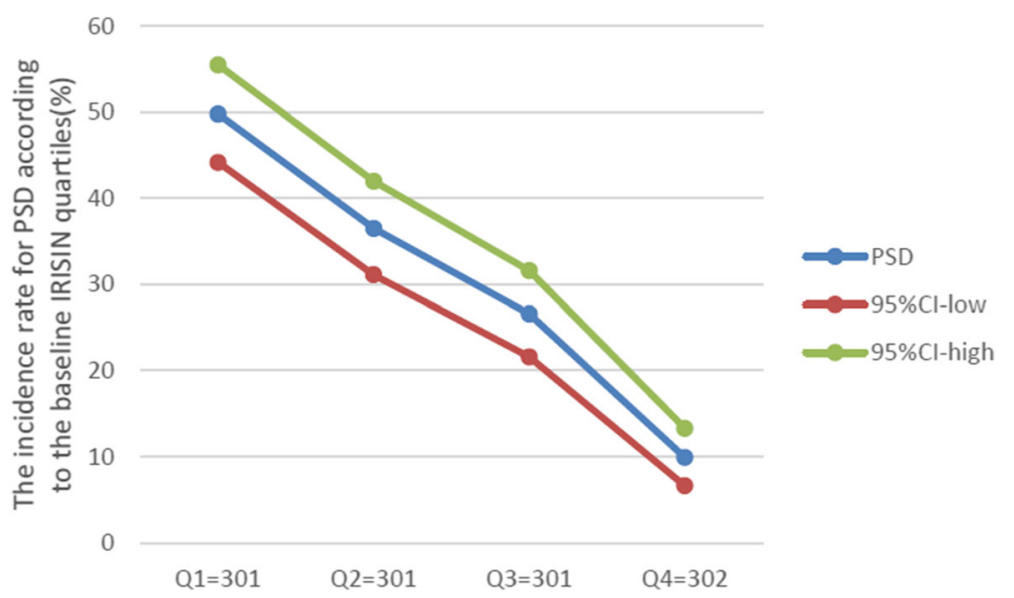

Fig. 2 The incidence for PSD according to the baseline irisin quartiles. Serum levels of irisin in quartile $1(<71.1 \mathrm{ng} / \mathrm{mL})$, quartile $2(71.1-95.9 \mathrm{ng} / \mathrm{mL})$, quartile 3 (96.0-144.0 ng/mL), and quartile 4 (> $144.0 \mathrm{ng} / \mathrm{mL}$ ). PSD post-stroke depression

levels of irisin in Q1, Q2, and Q3 were associated with PSD and increased risk of PSD by $385 \%(\mathrm{OR}=4.85 ; 95 \%$ CI, 3.30-7.92), 179\% (OR, 2.79; 95\% CI, 1.82-4.37), and $82 \%$ (1.82; 1.13-2.92). The independent association of irisin with PSD was confirmed using the likelihood ratio test $(P=0.002)$. In a multivariate model using the $\mathrm{Q} 1$ of irisin vs. Q2-4 together with the clinical variables, the marker displayed prognostic information and increased risk of PSD by 75\% (OR for Q1, 1.75 [95\% CI, 1.15-2.65]).

Using ROC curves, irisin levels $>85 \mathrm{ng} / \mathrm{mL}$ at admission predicted the development of depression at 3 months with the highest sensitivity and specificity [67.6 and 70.7\%, respectively; area under the curve $(\mathrm{AUC})=0.74 ; 95 \% \mathrm{CI}$, 0.67-0.81] (Fig. 4). With an AUC of 0.74, irisin showed a significantly greater discriminatory ability to predict PSD as compared with Hs-CRP (AUC, 0.61; 95\% CI, 0.56-0.67; $P<0.001$ ), HCY (AUC, 0.66; 95\% CI, 0.59-0.72; $P=0.002$ ),

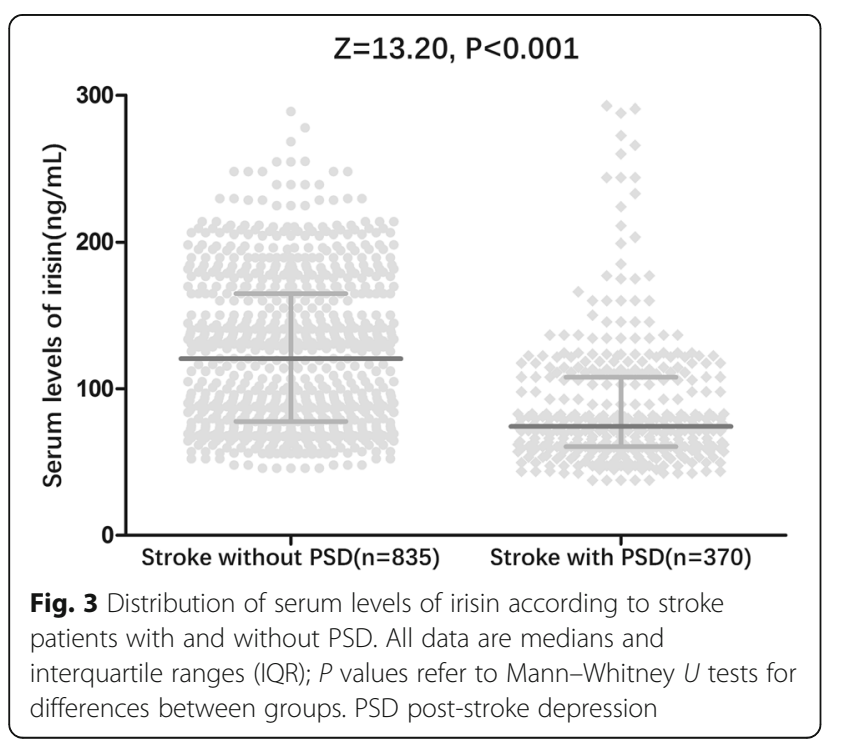

age (AUC, 0.59; 95\% CI, 0.53-0.65; $P<0.001$ ), NHISS score (AUC, 0.60 ; 95\% CI, 0.55-0.66; $P<0.001$ ), and serotonin $(0.69,0.59 ; 95 \% \mathrm{CI}, 0.63-0.76 ; P=0.009)$.

In addition, a model containing known risk factors plus irisin compared with a model containing known risk factors without irisin showed a greater discriminatory ability; the area under the curve (AUC) increased from 0.77 to 0.81 (95\% confidence interval [CI], 0.76-0.86). A significant difference in the AUC between the clinical variables alone and the addition of irisin level was observed (difference, 0.04 [95\% CI, 0.03-0.05]; $P=0.02$ ) (Table 3). The NRI statistic showed that the addition of irisin to established risk factors significantly increased the correct reclassification of depressive patients and non-depressive patients $(P=0.003)$. The IDI statistic also found that the irisin level significantly increased

Table 2 Odds ratios for PSD according to irisin quartiles at admission

\begin{tabular}{llll}
\hline $\begin{array}{l}\text { Irisin } \\
\text { quartiles }^{\ddagger}\end{array}$ & PSD, $N$ & $\begin{array}{l}\text { Unadjusted OR } \\
(95 \% \mathrm{Cl})^{\xi}\end{array}$ & $\begin{array}{l}\text { Adjusted OR } \\
(95 \% \mathrm{Cl})^{* \varepsilon}\end{array}$ \\
\hline $\mathrm{Q} 1, N=301$ & 150 & $9.01(5.80-13.98)$ & $4.85(3.30-7.92)$ \\
$\mathrm{Q} 2, N=301$ & 110 & $4.96(3.19-7.73)$ & $2.79(1.82-4.37)$ \\
$\mathrm{Q} 3, N=301$ & 80 & $3.28(2.08-5.18)$ & $1.82(1.13-2.92)$ \\
$\mathrm{Q} 4, N=302$ & 30 & References & References \\
\hline
\end{tabular}

OR odds ratio, $\mathrm{Cl}$ confidence interval, PSD post-stroke depression, NIHSS National Institutes of Health Stroke Scale, $m R S$ Modified Rankin Scale, Hs-CRP high-sensitivity C-reactive protein, $H C Y$ homocysteine, $B M I$ body mass index, $H D L-C$ high-density lipoprotein cholesterol, $L D L-C$ low-density lipoprotein cholesterol, TG triglyceride, TC total cholesterol, HOMA-IR homeostatic model of assessment insulin resistance, $I L-6$ interleukin- 6

${ }^{\ddagger}$ Serum levels of irisin in quartile $1(<71.1 \mathrm{ng} / \mathrm{mL})$, quartile $2(71.1-95.9 \mathrm{ng} /$ $\mathrm{mL})$, quartile $3(96.0-144.0 \mathrm{ng} / \mathrm{mL})$, and quartile $4(>144.0 \mathrm{ng} / \mathrm{mL})$

*Adjusted for age, sex, BMI, stroke etiology, the NIHSS score, infarct volume, pre-stroke and acute treatment, vascular risk factors, mRS at follow-up, family history of psychiatric disorders, lesion location, living with offspring, widowhood, HOMA-IR, blood levels of glucose, Hs-CRP, HCY, IL-6, TG, TC, HDL, LDL, and serotonin and irisin quartiles

$\varepsilon p$ value for the trend $<0.001$ 


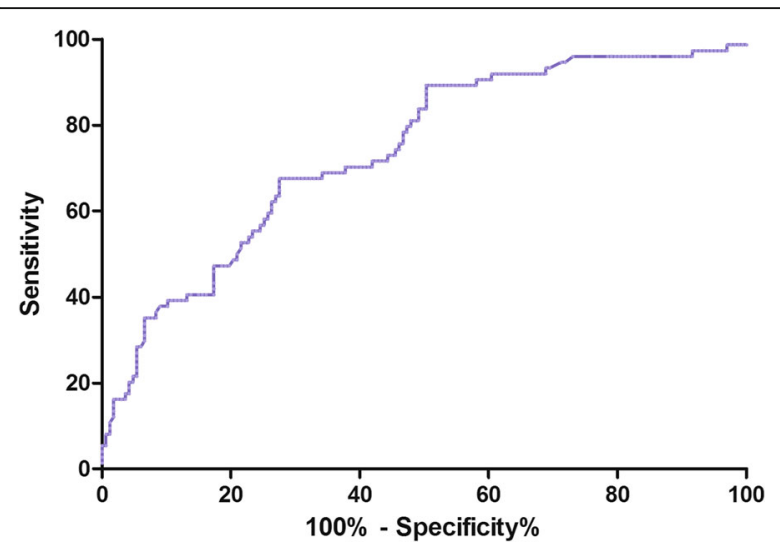

Fig. 4 Receiver operator characteristic curve demonstrating sensitivity as a function of 1-specificity for predicting the PSD within 6 months based on the serum irisin levels at admission. PSD post-stroke depression

discrimination between depressive patients and nondepressive patients $(P=0.01)$.

\section{Subgroup analysis}

In the subgroup of patients $(n=223)$ in whom physical activity evaluations were performed, 69 (30.9\%) patients were defined as depression patients. Physical activity in stroke patients with depression were lower compared with those in patients without depression [1.0 (IQR, 0.7-1.5) $\mathrm{kcal} / \mathrm{kg}$ day vs. 1.3 (IQR, 0.8-1.7) $\mathrm{kcal} / \mathrm{kg}$ day; $P=0.001$ ]. There was a positive correlation between levels of irisin and the physical activity $(r$ spearman $]=0.173, P=0.015)$. Physical activity in stroke patients with low level of irisin (Q1) were lower compared with those in patients with normal irisin (Q2-4) [0.8 (IQR, 0.5-1.2) kcal/kg day vs. 1 . 5 (IQR, $1.0-1.9$ ) $\mathrm{kcal} / \mathrm{kg}$ day; $P<0.001$ ]. In multivariate logistic regression analysis, we calculated the OR of irisin level to predict PSD after adjusted physical activity and other risk factors. Irisin was still an independent PSD predictor with an OR of 0.992 (95\% CI, 0.985-0.997; $P=0.003$ ) after adjustment for both physical activity and other risk factors. Similarly, in a multivariate model using the low (Q1) of irisin vs. normal (Q2-4) together with physical activity and other risk factors, the marker displayed prognostic information and increased risk of PSD by 63\% (OR for Q1, 1.63 [95\% CI, 1.12-2.57]).

\section{Discussion}

A review identified potential biopsychosocial variables associated with the relationship between obesity and depression [34]. This is the first study to date to assess concentration of irisin, a risk of obesity [35], at admission in relation to the development of PSD and to investigate its clinical utility in Chinese stroke patients. The findings of this study were as follows: (1) serum irisin levels in patients with depression were lower compared with those in patients without depression; (2) reduced serum levels of irisin were powerful biological markers of risk of developing PSD even after adjustment by variables, and thus, it may be used as a future therapeutic target in patients with ischemic stroke; and (3) irisin showed a significantly greater discriminatory ability to predict PSD as compared with other biomarkers, such as Hs-CRP, HCY, age, and serotonin.

Serotonin is strongly associated with the etiology of major depressive disorder and has been suggested as a key contributor to the regulation of mood and anxiety [36]. A previous study found that serotonin deficiency may be one of the factors leading to depression following stroke [37]. In this study, we also found that low levels of serotonin were useful biomarkers to predict PSD. However, the predictive efficiency was lower than irisin $(P=0.009)$. In addition, the predicted trends of irisin for PSD had not been changed, even after adjusted for serotonin in the multivariate analysis.

In this study, we found that serum levels of irisin in stroke patients were lower than in controls. Consistent with our results, Li et al. [38] reported that the plasma irisin concentration and intramuscular FNDC5 protein expression decreased after ischemic stroke. In this same study, the author confirmed that plasma irisin levels were negatively associated with brain infarct volume, the neurological deficit score, and plasma TNF- $\alpha$ and plasma IL-6 concentrations [38]. Similarly, our study suggested that irisin levels were negatively associated with infarct volume, the neurological deficit score, serum levels of CRP and IL-6. A previous study have shown that irisin

Table 3 Serum irisin concentrations at admission prediction of PSD with AUROC

\begin{tabular}{|c|c|c|c|c|c|c|}
\hline \multirow[t]{2}{*}{ PSD } & \multicolumn{4}{|c|}{ AUROC } & \multirow[b]{2}{*}{$\mathrm{NRI}(P)$} & \multirow[b]{2}{*}{$\mathrm{IDI}(P)$} \\
\hline & Irisin & Risk factors ${ }^{*}$ & Risk factors with IRISIN & Incremental area $(P)^{\ddagger}$ & & \\
\hline At admission & 0.74 & 0.77 & 0.81 & $0.04(0.02)$ & $0.13(0.003)$ & $0.06(0.01)$ \\
\hline \multicolumn{7}{|c|}{$\begin{array}{l}\text { PSD post-stroke depression, NIHSS National Institutes of Health Stroke Scale, } m R S \text { Modified Rankin Scale, } B M I \text { body mass index, } H s-C R P \text { high-sensitivity } C \text {-reactive } \\
\text { protein, } H C Y \text { homocysteine, } H D L-C \text { high-density lipoprotein cholesterol, } L D L-C \text { low-density lipoprotein cholesterol, } T G \text { triglyceride, } T C \text { total cholesterol, HOMA-IR } \\
\text { homeostatic model of assessment insulin resistance, IL-6 interleukin- } 6 \\
\text { ※ Established risk factors including age, sex, BMI, stroke etiology, the NIHSS score, infarct volume, pre-stroke and acute treatment, vascular risk factors, mRS at } \\
\text { follow-up, family history of psychiatric disorders, lesion location, living with offspring, widowhood, HOMA-IR, blood levels of glucose, Hs-CRP, HCY, IL-6, TG, TC, } \\
\text { HDL, LDL, and serotonin } \\
{ }^{\ddagger} \text { Comparison of AUROCs: established risk factors without irisin levels vs. established risk factors with irisin levels }\end{array}$} \\
\hline
\end{tabular}


levels were lower in myocardial infarction (MI) and coronary atherosclerosis diseases (CAD) implying that their production may depend on myocardial blood supply [39], while another study suggested that serum irisin level was an independent predictor of coronary artery severity in patients with stable coronary artery disease [40].

Although previous results have provided evidence that irisin may mediate some of the positive effects of training on body weight, insulin sensitivity, and glucose homeostasis, studies in humans have led to mixed results. Thus, some studies have reported a positive correlation between BMI, IR and circulating irisin levels [41, 42], or muscle FNDC5 mRNA expression $[4,12]$, while other studies have reported a negative correlation between BMI and circulating irisin levels $[10,43]$. In this study, there is a negative correlation between BMI, IR, and circulating irisin levels.

The mechanism by which irisin mediates the depressive effect on stroke is still unknown. A possible explanation is interplay with inflammation markers. Inflammation may represent a common mechanism of disease has been extended to include neuropsychiatric disorders including major depression [44]. In addition, a study provides evidence of an association between irisin and homocysteine, which may be due to nicotinamide metabolism [45]. Cheng et al. suggested that that elevated serum levels of Hs-CRP and HCY were associated with the risk of developing PSD [46]. However, in this study, irisin remained significantly associated with PSD even after adjustment for Hs-CRP or IL-6, suggesting that the effect of irisin on PSD was independent of inflammation.

Another possible explanation is interplay with other myokines, adipocytokines, or classical cytokines. Shan et al. [47] demonstrated an important interplay between irisin and myostatin, another myokine. A study suggested that the lowered adiponectin levels in depression are depression-specific and not explained by conventional low adiponectin-related factors [48]. Third, lower levels of irisin are independently associated with endothelial dysfunction. Endothelial dysfunction is associated with a greater depressive symptom score in a general elderly population [49]. Moreover, coronary endothelial dysfunction is associated with an increased risk of cerebrovascular events. Irisin alleviates endothelial dysfunction in type 2 diabetes partially via reducing oxidative/nitrative stresses through inhibiting signaling pathways implicating PKC- $\beta / \mathrm{NADPH}$ oxidase and $\mathrm{NF}-\kappa \mathrm{B} / \mathrm{iNOS}$ [15]. A study concludes that acute administration of irisin lowers blood pressure of SHRs by amelioration of endothelial dysfunction of the mesenteric artery through the AMPK-Akt-eNOS-NO signaling pathway [50]. Fourth, a previous study indicated that irisin serves as a novel approach to eliciting cardioprotection, which is associated with the improvement of mitochondrial function [2]. Della et al. [51] reported that complexes I, III, and IV of the mitochondrial respiratory chain were inhibited by chronic mild stress in the cerebral cortex and cerebellum, contributing to depressive-like behaviors in rats. Lastly, since the discovery of irisin, its levels have been associated with both cholesterol and steroid hormones, such as estradiol [41], which leads us to believe that it might be involved in the process of depression [52]. Hence, further studies should explore the molecular mechanism to elucidate direct irisin effects.

\section{Strengths and limitations}

The strengths of our study include the fact that it is a prospective multiethnic study with a relatively larger sample, making the results robust and generalizable. Furthermore, we collected data on a wide range of potentially confounding risk factors, allowing us to estimate the independent effect of irisin on PSD. Lastly, we chose a different strategy using the fourth quartiles, because we have found this strategy less sensitive to other factors that might influence the relatively low concentrations.

The following limitations of our study must be taken into account. First, although the data suggested an inverse correlation of irisin with post-stroke depression, investigation of cause-effect and mechanisms might be a more interesting question. These studies help to analyze whether the change of circulating irisin was pathogenically involved in the development of PSD or just a mark. However, the cross-sectional nature of the study precludes us to draw any conclusion on the role of irisin in the development of PSD, and therefore, no conclusion regarding cause-effect and mechanism relationships can be made. Therefore, the impact of irisin on future depression outcomes should be clarified in the future study. Second, irisin is usually measured by ELISA, but the quantification varies greatly between the kits. These differences probably come from the variety in the irisin epitopes being targeted for measurement by the manufacturing companies. Interestingly, two recent reports challenged the putative presence of irisin in human plasma [53]. Third, in the present study, there was no information in irisin levels according to regular exercise. However, concentrations of irisin increased significantly after endurance exercise training in both mice and humans [54]. Fourth, the dead patients had been excluded, who might suffer from depression. This approach may underestimate the incidence of depression. Fifth, PSD is a progressive complication; serum irisin levels in follow-up are valuable. However, in this study, only blood samples at admission were collected. Without serial measurement of the circulating irisin, this study yielded no data regarding when and how long biomarkers were reduced in these patients. Additionally, it should be investigated whether serial irisin testing further improves the risk stratification of PSD patients. Finally, all participants to the present study were Chinese Han, and whether these observations can also be 
extended to other ethnic groups with different body composition remains to be determined.

\section{Conclusions}

The present study suggested that reduced serum levels of irisin were powerful biological markers of risk of developing PSD even after adjustment by variables. Further studies are necessary to confirm this association, which may open the way to the proposal of new therapeutic options.

\section{Abbreviations}

AIS: Acute ischemic stroke; AUC: Area under the curve; BMI: Body mass index; CUS: Chronic unpredictable stress; CV: Coefficients of variation; DWI: Diffusion-weighted imaging; ELISA: Enzyme-linked immunosorbent assay; FNDC5: Fibronectin type III domain containing 5; HAM-D: Hamilton depression rating scale; HCY: Homocysteine; HDL-C: High-density lipoprotein cholesterol; HS-CRP: High-sensitivity C-reactive protein; IDI: Integrated discrimination improvement; IR: Insulin resistance; MRI: Magnetic resonance imaging; mRS: Modified Rankin Scale; NIHSS: National Institute of Health Stroke Scale; NRI: Net reclassification improvement; OR: Odds ratios; PSD: Post-stroke depression; ROC: Receiver operating characteristic curves; TC: Total cholesterol; TG: Triglycerides; TOAST: Trial of Org 10172 in Acute Stroke Treatment
\end{abstract}

\section{Acknowledgements}

All authors have contributed significantly, and all authors are in agreement with the content of the manuscript. We are grateful to the Department of Neurology and Emergency Department; the nurses, physicians, and patients who participated in our study; and the staff of the central laboratory of the hospital. We thank Pro Xiao-Lin Yang (Peking Union Medical College) for the statistical advice and assistance with the qualitative measures used in this study. We also thank Dr. Jian-lei Cao (Zhongnan Hospital Affiliated to Wuhan University) for the blood samples and clinical information collected.

\section{Role of the sponsor}

The funding organizations had no role in the design and concept of the study - the collection, management, analysis, and interpretation of the data or the preparation, review, or approval of the manuscript.

\section{Funding}

This study was supported by grants from Shandong Province Science and Technology Development Plan Project (No. 2015GGB14296), Shandong Province Key Research and Development Projects (No. 2016GSF201229), National Natural Science Foundation of China (No. 81671175), and CAMS Innovation Fund for Medical Science (No. 2017-I2M-1-016).

\section{Availability of data and materials}

Please contact the corresponding author for data requests.

\section{Authors' contributions}

ZJZ had full access to all of the data in the study and takes responsibility for the integrity of the data and the accuracy of the data analysis. TWJ, QHC, LQ, LXM, ZJZ, and ZXW contributed to the study concept and design. TWJ, QHC, and LXM contributed to the acquisition of the data. LQ, ZJZ, and ZXW carried out the analysis and interpretation of the data. TWJ and QHC contributed in the drafting of the manuscript. LQ, LXM, ZJZ, and ZXW are responsible for the critical revision of the manuscript for important intellectual content. TWJ, OHC, LO and LXM are responsible for the administrative, technical, or material support. LQ and ZXW obtained the funding. ZX supervised the study. All authors read and approved the final manuscript.

\section{Ethics approval and consent to participate}

Written informed consents were obtained from all patients, and this study conformed to the principles of the Declaration of Helsinki and was approved by the investigational review board of the Weifang Medical University. (Trial registration: ChiCTR-OPC-17013501. Retrospectively registered 23 September 2017).

\section{Competing interests}

The authors declare that they have no competing interests.

\section{Publisher's Note}

Springer Nature remains neutral with regard to jurisdictional claims in published maps and institutional affiliations.

\section{Author details}

'Institute of Radiation Medicine, China Academy of Medical Science and Peking Union Medical College, Tianjin, China. ${ }^{2}$ Department of Neurosurgery, Beijing Tiantan Hospital of Capital Medical University, Beijing, China. ${ }^{3}$ Center for Translational Medicine, Institutes of Stroke, Weifang Medical University, Weifang, China. ${ }^{4}$ Tianjin, China. ${ }^{5}$ Beijing, China.

Received: 19 February 2018 Accepted: 24 April 2018

Published online: 02 May 2018

References

1. Mathers CD, Boerma T, Ma FD. Global and regional causes of death. Br Med Bull. 2009:92(1):7-32.

2. Cheng SY, Zhao YD, Li J, Chen XY, Wang RD, Zeng JW. Plasma levels of glutamate during stroke is associated with development of post-stroke depression. Psychoneuroendocrinology. 2014;47:126-35.

3. Hadidi N, Treat-Jacobson DJ, Lindquist R. Poststroke depression and functional outcome: a critical review of literature. Heart Lung. 2009;38(2):151-62.

4. Boström P, Wu J, Jedrychowski MP, Korde A, Ye L, Lo JC. A PGC1-adependent myokine that drives brown-fat-like development of white fat and thermogenesis. Nature. 2012;481:463-8.

5. Huh JY, Mougios V, Kabasakalis A, Fatouros I, Siopi A, Douroudos II. Exerciseinduced irisin secretion is independent of age or fitness level and increased irisin may directly modulate muscle metabolism through AMPK activation. J Clin Endocrinol Metab. 2014;99(11):E2154-61.

6. Ferrer-Martínez A, Ruiz-Lozano P, Chien KR. Mouse PeP: a novel peroxisomal protein linked to myoblast differentiation and development. Dev Dyn. 2002; 224:154-67.

7. Xin C, Liu J, Zhang J, Zhu D, Wang H, Xiong L. Irisin improves fatty acid oxidation and glucose utilization in type 2 diabetes by regulating the AMPK signaling pathway. Int J Obes. 2016;40(3):443.

8. Lee HJ, Lee JO, Kim N, Kim JK, Kim HI, Lee YW. Irisin, a novel myokine, regulates glucose uptake in skeletal muscle cells via AMPK. Mol Endocrinol. 2015;29(6):873-81.

9. Rodríguez-Carmona A, Fontán MP, Alvarellos SS. Serum levels of the adipomyokine irisin in patients with chronic kidney disease. Nefrología. 2016:36(5):496-502.

10. Moreno-Navarrete JM, Ortega F, Serrano M, Guerra E, Pardo G, Tinahones F. Irisin is expressed and produced by human muscle and adipose tissue in association with obesity and insulin resistance. J Clin Endocrinol Metab. 2013:98:E769-78.

11. Crujeiras AB, Zulet MA, Lopez-Legarrea P, de la Iglesia R, Pardo M, Carreira $M C$. Association between circulating irisin levels and the promotion of insulin resistance during the weight maintenance period after a dietary weight-lowering programin obese patients. Metabolism. 2014;63:520-31.

12. Choi YK, Kim MK, Bae KH, Seo HA, Jeong JY, Lee WK. Serum irisin levels in new-onset type 2 diabetes. Diabetes Res Clin Pract. 2013;100:96-101.

13. Zhang W, Chang L, Zhang C, Zhang R, Li Z, Chai B. Irisin: a myokine with locomotor activity. Neurosci Lett. 2015;595:7-11.

14. Brondani LA, Boelter G, Assmann TS, Leitao CB, Canani LH, Crispim D. Irisinencoding gene (FNDC5) variant is associated with changes in blood pressure and lipid profile in type 2 diabetic women but not in men. Metabolism. 2015;64:952-7.

15. Zhu D, Wang H, Zhang J, Zhang X, Xin C, Zhang F. Irisin improves endothelial function in type 2 diabetes through reducing oxidative/nitrative stresses. J Mol Cell Cardiol. 2015;87:138-47.

16. Han F, Zhang S, Hou N, Wang D, Sun X. Irisin improves endothelial function in obese mice through the AMPK-eNOS pathway. Am J Physiol Heart Circ Physiol. 2015;309:H1501-8.

17. Lu J, Xiang G, Liu M, Mei W, Xiang L, Dong J. Irisin protects against endothelial injury and ameliorates atherosclerosis in apolipoprotein E-null diabetic mice. Atherosclerosis. 2015;243:438-48.

18. Chen J, Huang Y, Gusdon AM, Qu S. Irisin: a new molecular marker and target in metabolic disorder. Lipids Health Dis. 2015;14(1):2. 
19. Skilton MR, Moulin P, Terra JL, Bonnet F. Associations between anxiety, depression, and the metabolic syndrome. Biol Psychiatry. 2007;62(11):1251-7.

20. Vogelzangs N, Suthers K, Ferrucci L, Simonsick EM, Ble A, Schrager M. Hypercortisolemic depression is associated with the metabolic syndrome in late-life. Psychoneuroendocrinology. 2007;32(2):151-9.

21. Roca-Rivada A, Castelao C, Senin LL, Landrove MO, Baltar J, Belen CA. FNDC5/irisin is not only a myokine but also an adipokine. PLoS One. 2013;8: e60563.

22. Carvalho AF, Rocha DQ, Mclntyre RS, Mesquita LM, Köhler CA, Hyphantis TN. Adipokines as emerging depression biomarkers: a systematic review and meta-analysis. J Psychiatr Res. 2014;59:28-37.

23. Wang S, Pan J. Irisin ameliorates depressive-like behaviors in rats by regulating energy metabolism. Biochem Biophys Res Commun. 2016;474(1):22-8.

24. Schuch FB, Vancampfort D, Richards J, et al. Exercise as a treatment for depression: a meta-analysis adjusting for publication bias. J Psychiatr Res. 2016;77:42-51.

25. Wrann CD. FNDC5/irisin - their role in the nervous system and as a mediator for beneficial effects of exercise on the brain. Brain Plasticity. 2015; 1(1):55-61.

26. Wu H, Guo $P$, Jin Z, et al. Serum levels of irisin predict short-term outcomes in ischemic stroke. Cytokine. 2018; https://doi.org/10.1016/j.cyto.2018.02.017.

27. Brott T, Adams HP Jr, Olinger CP, Marler JR, Barsanl WG, Biller J. Measurements of acute cerebral infarction: a clinical examination scale. Stroke. 1989;20:864-70.

28. Sims JR, Gharai LR, Schaefer PW, Vangel M, Rosenthal ES. ABC/2 for rapid clinical estimate of infarct, perfusion, and mismatch volumes. Neurology. 2009;72:2104-10

29. DATA D. Structured clinical interview for DSM-IV axis I disorders. Washington: American Psychiatric Press; 1997.

30. Hamilton M. A rating scale for depression. J Neurol Neurosurg Psychiatry. 1960;23(1):56-62.

31. Bonita RR. Modification of Rankin scale: recovery of motor function after stroke. Stroke. 1988;19:1497-500.

32. Nowrouzi-Kia B, Li AKC, Nguyen C, et al. Heart disease and occupational risk factors in the Canadian population: an exploratory study using the Canadian community health survey. Safety Health Work. 2017; https://doi. org/10.1016/j.shaw.2017.07.008.

33. Pencina MJ, D'Agostino RB Sr, D'Agostino RB Jr, Vasan RS. Evaluating the added predictive ability of a new marker: from area under the ROC curve to reclassification and beyond. Stat Med. 2008:27:157-72.

34. Preiss K, Brennan L, Clarke D. A systematic review of variables associated with the relationship between obesity and depression. Obes Rev. 2013; 14(11):906-18.

35. Jang HB, Kim HJ, Kang JH, Park SI, Park KH, Lee HJ. Association of circulating irisin levels with metabolic and metabolite profiles of Korean adolescents. Metabolism. 2017;73:100-8.

36. Robson MJ, Quinlan MA, Blakely RD. Immune system activation and depression: roles of serotonin in the central nervous system and periphery. ACS Chem Neurosci. 2017:8(5):932-42.

37. Gao H, Zhu H, Zhang Y, et al. Reduction of cerebrospinal fluid and plasma serotonin in patients with post-stroke depression: a preliminary report. Clinical \& Investigative Medicine. 2008;31(6):351-6.

38. Li DJ, Li YH, Yuan HB, Qu LF, Wang P. The novel exercise-induced hormone irisin protects against neuronal injury via activation of the Akt and ERK1/2 signaling pathways and contributes to the neuroprotection of physical exercise in cerebral ischemia. Metabolism. 2017;68:31-42.

39. Anastasilakis AD, Koulaxis D, Kefala N, Polyzos SA, Upadhyay J, Pagkalidou E. Circulating irisin levels are lower in patients with either stable coronary artery disease (CAD) or myocardial infarction (MI) versus healthy controls, whereas follistatin and activin A levels are higher and can discriminate MI from CAD with similar to CK-MB accuracy. Metabolism. 2017;73:1-8.

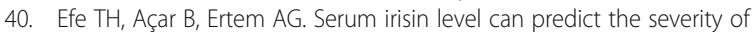
coronary artery disease in patients with stable angina. Korean Circ J. 2017; 47(1):44-9.

41. Huh JY, Panagiotou G, Mougios V, Brinkoetter M, Vamvini MT, Schneider BE. FNDC5 and irisin in humans: I. Predictors of circulating concentrations in serum and plasma and II. mRNA expression and circulating concentrations in response to weight loss and exercise. Metab Clin Exp. 2012;61:1725-38.

42. Park KH, Zaichenko L, Brinkoetter M, Thakkar B, Sahin-Efe A, Joung KE. Circulating irisin in relation to insulin resistance and the metabolic syndrome. J Clin Endocrinol Metab. 2013;98:4899-907.
43. Hwang YC, Jeon WS, Park CY, Youn BS. The ratio of skeletal muscle mass to visceral fat area is a main determinant linking circulating irisin to metabolic phenotype. Cardiovasc Diabetol. 2016;15:9.

44. Miller $\mathrm{AH}$, Maletic $\mathrm{V}$, Raison $\mathrm{CL}$. Inflammation and its discontents: the role of cytokines in the pathophysiology of major depression. Biol Psychiatry. 2009; 65(9):732-41.

45. Alis R, Sanchis-Gomar F, Pareja-Galeano H, Hernández-Mijares A, Romagnoli M, Víctor VM. Association between irisin and homocysteine in euglycemic and diabetic subjects. Clin Biochem. 2014;47(18):333-5.

46. Cheng LS, Tu WJ, Shen Y, Zhang LJ, Ji K. Combination of high-sensitivity Creactive protein and homocysteine predicts the post-stroke depression in patients with ischemic stroke. Mol Neurobiol. 2018;55(4):2952-8.

47. Shan T, Liang $X, B i P$, Kuang $S$. Myostatin knockout drives browning of white adipose tissue through activating the AMPK-PGC1alpha-Fndc5 pathway in muscle. FASEB J. 2013;27:1981-9.

48. Lehto SM, Huotari A, Niskanen L, Tolmunen T, Koivumaa-Honkanen H, Honkalampi K. Serum adiponectin and resistin levels in major depressive disorder. Acta Psychiatr Scand. 2010;121(3):209-15.

49. Van Sloten TT, Schram MT, Adriaanse MC, Dekker JM, Nijpels G, Teerlink T. Endothelial dysfunction is associated with a greater depressive symptom score in a general elderly population: the Hoorn study. Psychol Med. 2014; 44(7):1403-16.

50. Fu J, Han Y, Wang J, Liu Y, Zheng S, Zhou L. Irisin lowers blood pressure by improvement of endothelial dysfunction via AMPK-Akt-eNOS-NO pathway in the spontaneously hypertensive rat. J Am Heart Assoc. 2016;5(11): e003433.

51. Della FP, Abelaira HM, Réus GZ, Ribeiro KF, Antunes AR, Scaini G. Tianeptine treatment induces antidepressive-like effects and alters BDNF and energy metabolism in the brain of rats. Behav Brain Res. 2012;233(2):526-35.

52. Balzer BWR, Duke SA, Hawke Cl. The effects of estradiol on mood and behavior in human female adolescents: a systematic review. Eur J Pediatr. 2015;174(3):289-98.

53. Raschke S, Elsen M, Gassenhuber H, Sommerfeld M, Schwahn U, Brockmann B. Evidence against a beneficial effect of irisin in humans. PLoS One. 2013;8: e73680.

54. Sanchis-Gomar F, Perez-Quilis C. Irisinemia: a novel concept to coin in clinical medicine? Ann Nutr Metab. 2013:63(1-2):60-1.

\section{Ready to submit your research? Choose BMC and benefit from:}

- fast, convenient online submission

- thorough peer review by experienced researchers in your field

- rapid publication on acceptance

- support for research data, including large and complex data types

- gold Open Access which fosters wider collaboration and increased citations

- maximum visibility for your research: over $100 \mathrm{M}$ website views per year

At BMC, research is always in progress.

Learn more biomedcentral.com/submissions 